\title{
Plantas hospedantes de Bemisia tabaci (Gennadius) (Hemíptera: Aleyrodidae), en áreas colindantes al cultivo de tomate, en la región este de Panamá
}

\author{
Hosts plants of Bemisia tabaci (Gennadius) (Hemíptera: Aleyrodidae), \\ in adjacent areas of the tomato crop, in the east region of Panama
}

Bruno Zachrisson ${ }^{*}$, José Ángel Herrera-Vásquez ${ }^{1}$, Juan Bernal ${ }^{2}$

\begin{abstract}
RESUMEN
Las plantas hospedantes de huevos y ninfas de Bemisia tabaci (Gennadius), totalizaron 16 especies agrupadas en 11 familias, las que fueron muestreadas y recolectadas en áreas adyacentes a las parcelas de producción de tomate. Este estudio se realizó en la localidad de "La Mesa de San Martín", en la región este de Panamá, mediante evaluaciones trimestrales realizadas entre mayo de 2013 y junio de 2014. Se realizaron muestreos aleatorios que constaron de 15 puntos de recolección de $1 \mathrm{~m}^{2}$, evaluándose el número de huevos y ninfas de este insecto vector de begomovirus, en cinco hojas o foliolos en los estratos superior, medio e inferior de las plantas. La superficie total muestreada por hoja o foliolo de la planta hospedante fue de $1.000 \mathrm{~cm}^{2}$. La mayor incidencia de huevos y ninfas de B. tabaci se registró en Guazuma ulmifolia Lam., Jatropha gossypifolia L., Solanum torvum Sw., Jatropha urens L. y Physalis angulata L. Se discutió el papel de las plantas hospedantes de B. tabaci en la diseminación de begomovirus y el manejo de esta interacción biótica en las áreas de producción de tomate.
\end{abstract}

Palabras claves: hospedero, mosca blanca, Solanum lycopersicum.

\begin{abstract}
The hosts plants of eggs and nymphs of Bemisia tabaci (Gennadius), totaled 16 species grouped in 11 families, which were sampled and collected in adjacent areas to the plots of tomato production. This study was conducted in the locality of "La Mesa de San Martín", in the East region of Panama, through quarterly assessments carried out between May of 2013 and June 2014. Is performed random sampling that consisted of 15 points of $1 \mathrm{~m}^{2}$, assessing the numbers of eggs and the nymphs of this insect vector of begomovirus, in five leaves or leaflets in the upper, medium and lower level of the plants. The total area sampled by leaf or leaflet of the host plant was 1,000 $\mathrm{cm}^{2}$. The greater incidence of eggs and nymphs of B. tabaci is recorded in Guazuma ulmifolia Lam., Jatropha gossypifolia $L$., Solanum torvum $S w$., Jatropha urens $L$. and Physalis angulata $L$. Discussed the role of the host plants of B. tabaci in the begomovirus spread and the management of this biotic interaction in the areas of tomato production.
\end{abstract}

Key words: host, Solanum lycopersicum, whitefly.

\section{Introducción}

En Panamá, la superficie total destinada al cultivo de tomate es de 799 ha, con una producción de $20.097 \mathrm{t}$ (FAO, 2016), la que se concentra en las provincias de Los Santos (tomate industrial) y Chiriquí (tomate de mesa), en la región central y occidental de este país, respectivamente (INEC, 2016). Sin embargo, el área destinada al cultivo de este rubro agrícola se ha expandido a otras regiones agroclimáticas, que incluye a la región este de Panamá (INEC, 2016). En Panamá, la alta incidencia de Bemisia tabaci (Gennadius) está asociada a la transmisión de diferentes especies de los géneros begomovirus y torradovirus (Engel et al., 1998; Herrera-Vásquez et al., 2009, 2015, 2016; Herrera-Vásquez y Cortés, 2015). La interacción biótica entre este insecto vector y los virus que transmite, se agrava en función del elevado número de plantas hospedantes

\footnotetext{
1 Instituto de Investigación Agropecuaria de Panamá (IDIAP). Panamá, Panamá.

2 Universidad Autónoma de Chiriquí (UNACHI), Facultad de Ciencias Naturales y Exactas, Escuela de Biología, Chiriquí, Panamá.

* Autor para correspondencia: bruno.zachrissons@idiap.gob.pa; bazsalam@gmail.com
}

Fecha de Recepción: 27 Enero, 2017.

Fecha de Aceptación: 3 Febrero, 2017.

DOI: $10.4067 / \mathrm{S} 0718-34292017005000019$ 
reportadas en áreas próximas a cultivos de tomate (Morales, 2006). Aproximadamente, más de 600 especies pertenecientes a 74 familias de plantas, en agroecosistemas tropicales y subtropicales, presentan las diferentes fases de desarrollo de B. tabaci (Naik et al., 2003; Cuéllar y Morales, 2006). La capacidad de adaptación del grupo Medio Oriente-Asia Menor 1 (Middle East Asia Minor 1, MEAM1, anteriormente referida como biotipo B) de B. tabaci (De Barro et al., 2011; Lee et al., 2013) a este elevado número de especies vegetales, dificulta su manejo (Morales, 2006). Esta condición biológica favorece la diseminación de begomovirus, aunado a la capacidad del vector del grupo MEAM1 (Cuéllar y Morales, 2006). En Panamá, la predominancia de este grupo de B. tabaci y el desplazamiento del grupo del Nuevo Mundo (New World, NW, anteriormente referido como biotipo A) por el grupo MEAM1 (De Barro et al., 2011; Lee et al., 2013), es consecuencia de un proceso natural confirmado en otras regiones hortícolas de América Latina (Morales, 2006; Cuéllar y Morales, 2006). El daño directo causado por B. tabaci provoca desórdenes fisiológicos en la planta, entre estos la madurez irregular del fruto en el cultivo del tomate (Cuéllar y Morales, 2006). Indirectamente, los begomovirus transmitidos por este insecto vector afectan tanto a plantas cultivadas como no cultivadas, ocasionando pérdidas económicas significativas (Cuéllar y Morales, 2006). Diversas especies de malezas, entre estas Jatropha gossypifolia L. y Sida rhombifolia L., se consideran hospedantes de begomovirus (Morales, 2006). De igual forma, en áreas próximas a parcelas de producción de tomate, se han registrado especies vegetales como Amaranthus spinosus L., Amaranthus viridis L., Ageratum conyzoides L. y Bidens pilosa L., que hospedan y favorecen la diseminación de begomovirus, en presencia de B. tabaci (Silva et al., 2010). Este estudio proporciona las bases para el manejo de este agroecosistema, a partir del reporte del complejo de especies de plantas, consideradas hospedantes de B. tabaci con capacidad de diseminación de begomovirus, en la región este de Panamá.

\section{Materiales y Métodos}

La recolección de plantas hospedantes de B. tabaci se realizó en la localidad de "La Mesa de San Martín”, provincia de Panamá, Panamá ( $9^{\circ} 12^{\prime} \mathrm{N}$ y $79^{\circ} 15^{\prime} \mathrm{O}$ ), entre los meses de mayo de 2013 y junio de 2014. Se realizaron evaluaciones periódicas trimestralmente en 15 puntos de colecta de $1 \mathrm{~m}^{2}$, seleccionados aleatoriamente en los bordes de ocho parcelas de tomate. El conteo de huevos y ninfas de B. tabaci se realizó en cinco hojas o foliolos en cada planta, distribuidos en los estratos superior, medio e inferior, considerando un total de $1.000 \mathrm{~cm}^{2}$ de superficie foliar en cada muestra. Posteriormente, se recolectaron ninfas de $4^{\circ}$ instar y se transfirieron a viales de vidrio de $10 \mathrm{ml}$ conteniendo alcohol al $70 \%$, para su montaje e identificación, en el Laboratorio de Entomología del Centro de Investigación Agropecuaria Oriental (CIAOr) del Instituto de Investigación Agropecuaria de Panamá (IDIAP) (Panamá), de acuerdo con la clave taxonómica propuesta por Caballero (1994). Los especialistas del Herbario de la Universidad de Panamá, confirmaron las especies de plantas hospedantes de $B$. tabaci. La relación porcentual entre el promedio de huevos y ninfas de B. tabaci por planta hospedante, durante el período de evaluación y el total de ejemplares recolectados en cada fase de desarrollo, permitió determinar la tasa de infestación.

\section{Resultados y Discusión}

La presencia de huevos y ninfas de $B$. tabaci en las plantas recolectadas, confirman la condición de hospedantes para este insecto vector, denominados "hospedantes reproductivos" por Morales (2006) (Tabla 1), con capacidad de hospedar begomovirus. Las áreas colindantes a las parcelas de tomate muestreadas en la localidad de "La Mesa de San Martín”, presentaron 16 especies consideradas plantas no cultivadas, agrupadas en 11 familias que proporcionaron condiciones ideales para el desarrollo biológico de huevos y ninfas de $B$. tabaci (Tabla 1). Las especies correspondientes a las familias Malvaceae, Euphorbiaceae y Solanaceae, presentaron tasas elevadas de infestación de huevos y ninfas del insecto, durante el período de estudio (Tabla 1). De esta forma, Guazuma ulmifolia Lam. (Malvaceae), J. gossypifolia L. y Jatropha urens L. (Euphorbiaceae), Physalis angulata L. y Solanum torvum Sw. (Solanaceae), fueron las especies más representativas de las plantas hospedantes de B. tabaci (Tabla 1). La incidencia de las fases inmaduras de B. tabaci en este complejo de especies vegetales y su capacidad de transmisión de begomovirus en el cultivo de tomate, confirmaron 
Tabla 1. Tasa de infestación de huevos y ninfas de Bemisia tabaci (Gennadius) (Hemíptera: Aleyrodidae), recolectados en plantas hospedantes de áreas adyacentes a parcelas de producción de tomate (Solanum lycopersicum), en la localidad de "La Mesa de San Martín", provincia de Panamá, Panamá.

\begin{tabular}{lllcc}
\hline \multicolumn{1}{c}{ Familia } & \multicolumn{1}{c}{ Especie } & Nombre común & Huevos $(\%)^{1}$ & Ninfas $(\%)$ \\
\hline Amaranthaceae & Amaranthus dubius Mart. & Bledo & 2,05 & 1,56 \\
Asteraceae & Baltimora recta L. & Flor amarilla & 1,68 & 1,43 \\
Bixaceae & Bixa orellana L. & Achiote & 0,39 & 0,27 \\
Boraginaceae & Heliotropum indicum L. & Cola de alacrán & 1,48 & 1,16 \\
Cucurbitaceae & Momordica charantia L. & Balsamino & 0,31 & 0,27 \\
Fabaceae & Crotalaria retusa L. & Crotalaria & 0,44 & 0,41 \\
Euphorbiaceae & Euphorbia heterophila L. & Flor de pascua & 2,37 & 1,54 \\
& Jatropha gossypifolia L. & Frailecillo & 17,25 & 15,36 \\
Lamiaceae & Jatropha urens L. & Ortiga & 11,58 & 14,62 \\
Malvaceae & Hyptis capitata Jacq. & Suspiro de monte & 0,96 & 0,62 \\
& Malachra alceifolia Jacq. & Malva & 6,22 & 3,67 \\
& Sida rhombifolia L. & Escobilla & 0,53 & 0,58 \\
Portulacaceae & Guazuma ulmifolia Lam. & Guásimo & 28,23 & 31,14 \\
Solanaceae & Portulaca oleraceae L. & Verdolaga & 0,84 & 0,79 \\
& Physalis angulata L. & Topetón & 9,38 & 12,85 \\
& Solanum torvum Sw. & Araña gato & 16,29 & 13,73 \\
\hline
\end{tabular}

${ }^{1}$ Tasa de infestación de huevos y ninfas de Bemisia tabaci, expresada en porcentaje (\%).

la relevancia de esta interacción biótica (Bezerra et al., 2004; Silva et al., 2010). Estudios realizados por Morales (2006), reafirmaron la diseminación de begomovirus transmitida por B. tabaci, mediante la alimentación de Malva sp. y S. rhombifolia, en diversas zonas de producción agrícola. A partir de 1991, la presencia de begomovirus se consideró un grave problema para la producción de tomate industrial, debido al incremento de las poblaciones de su vector B. tabaci, por la aparición del grupo MEAM1 (biotipo B) de este insecto, mucho más agresivo que el grupo NW (biotipo A) (Zachrisson y Poveda, 1992; Engel et al., 1998). La presencia de begomovirus en siete especies de malezas, entre estas Euphorbia heterophylla L. (Euphorbiaceae), Herissanthia crispa (L.) Brizicky, Sida acuta Burm.f. y S. rhombifolia L. (Malvaceae), Corchorus orinocensis Kunth (Tiliaceae) y Rhynchosia minima (L.) DC. (Fabaceae), y Eleutheranthera ruderalis (Sw.) Sch.Bip. (Asteraceae), fue reportada en la proximidad de parcelas de producción de tomate, en la provincia de Los Santos, Panamá (Herrera-Vásquez, comunicación personal). Por lo tanto, el papel de plantas hospedantes en el desarrollo biológico y reproductivo de $B$. tabaci, es fundamental en la planificación e implementación de estrategias de manejo, con la finalidad de reducir la diseminación de begomovirus en otras zonas de producción de este rubro agrícola, en Panamá.

\section{Conclusiones}

La presencia de huevos y ninfas de $B$. tabaci, en especies de plantas hospedantes "no cultivadas" de diferentes familias, indican el grado de adaptación de este insecto vector de begomovirus, en áreas colindantes a parcelas de producción de tomate. La calidad nutricional de las especies hospedantes, contribuyen al desarrollo biológico de este insecto vector, favoreciendo el incremento de la población de este insecto vector, condición que puede propiciar la diseminación de la virosis, por lo que es relevante se considere la interacción "insecto vector-begomovirusplanta hospedante (no cultivada)", en la planificación e implementación de programas de manejo integrado de plagas (MIP), en este ecosistema agrícola.

\section{Agradecimientos}

Los autores agradecen al Sistema Nacional de Investigación (SNI) de la Secretaría Nacional de Ciencia Tecnología e Innovación (SENACYT), por el apoyo prestado en las diferentes etapas de esta investigación. 


\section{Literatura Citada}

Bezerra, M.S.; Oliveira, M.R.V.; Vasconcelos, S.D.

2004. Does the presence of weeds affect Bemisia tabaci (Gennadius) (Hemíptera: Aleyrodidae) infestation on tomato plants in semi-arid agro-ecosystem? Neotropical Entomology, 33 (6): 769-775.

Caballero, R.

1994. Clave de campo para inmaduros de moscas blancas en Centroamérica (Homoptera: Aleyrodidade). Escuela Agrícola Panamericana. Departamento de Protección Vegetal, Escuela Agrícola Panamericana, Zamorano, Honduras, $4 \mathrm{p}$.

Cuéllar, M.E.; Morales, F.J.

2006. La mosca blanca Bemisia tabaci (Gennadius) como plaga y vectora de virus en frijol común (Phaseolus vulgaris L.). Revista Colombiana de Entomología, 32 (1): 1-9.

De Barro, P.J.; Liu, S.S.; Boykin, L.M.; Dinsdale, A.B. 2011. Bemisia tabaci: a statement of species status. Annual Review of Entomology, 56: 1-19.

Engel, M.; Fernández, O.; Jeske, H.; Frischmuth, T. 1998. Molecular characterization of a new whiteflytransmissible bipartite geminivirus infecting tomato in FAO Panama. Journal of General Virology, 79: 2313-2317.

2016. Base de datos estadísticos de la Organización de las Naciones Unidas para la Alimentación y la Agricultura (FAO, por sus siglas en inglés). http://faostat3.fao.org/ faostat-gateway/go/to/download/Q/QC/E. Consultado: 22/ $\mathrm{dic} / 2016$.

Herrera-Vásquez, J.A.; Alfaro-Fernández, A.; Córdoba-Sellés, M.C.; Cebrián, M.C.; Font, M.I.; Jordá, C.

2009. First report of Tomato torrado virus infecting tomato in single and mixed infections with Cucumber mosaic virus in Panama. Plant Disease, 93 (2): 198.

Herrera-Vásquez, J.A.; Cortés, D.

2015. Presencia y distribución del virus del torrado del tomate en panamá. Ciencia Agropecuaria, 22: 16-31.
Herrera-Vásquez, J.A.; Ortega, D.; Romero, A.B.; Davino, S.; Mejía, L.C.; Panno, S.; Davino, M.

2016. Begomoviruses infecting tomato crops in Panama. Journal of Phytopathology, 164 (2): 102-113.

Herrera-Vásquez, J.A.; Ortega, D.; Romero, A.B.; Davino, S.; Mejía, L.C.; Panno, S.; Davino, M.

2015. First report of Tomato leaf curl sinaloa virus infecting tomato crops in Panama. New Disease Reports, 31: 30.

INEC.

2016. Instituto Nacional de Estadística y Censo, Contraloría General de la República de Panamá. https://www.contraloria. gob.pa/inec/Publicaciones/Publicaciones.aspx?ID_ SUBCATEGORIA=60\&ID_PUBLICACION $=479 \& I D \_$ IDIOMA=1\&ID_CATEGORIA=15. Consultado: 22/dic/2016.

Lee, W.; Park, J.; Lee, G.S.; Lee, S.; Akimoto, S-I.

2013. Taxonomic status of the Bemisia tabaci complex (Hemíptera: Aleyrodidae) and reassessment of the number of its constituent species. PLoS ONE, 8 (5): e63817 doi:10.1371/ journal.pone.0063817

Morales, F.J.

2006. History and current distribution of begomoviruses in Latin America. Advances in Virus Research, 67: 127-162.

Naik, S.; Muniyappa, V.; Colvin, J.

2003. Host preference and natural occurrence of Bemisia tabaci (Gennadius) on weed hosts - a vector of Tomato Leaf Curl Geminivirus Disease. Indian Journal of Agriculture Research, 37 (4): 253-258.

Silva, A.K.F.; Santos, C.D.G.; Nascimento, A.K.Q.

2010. Transmissão de begomovirus de plantas daninhas para tomateiros pela mosca-branca. Planta Daninha, 28 (3): 507-514.

Zachrisson, B.; Poveda, J.

1992. Las moscas blancas en Panamá. In: Hilje, L; Arboleada, O. (eds.). Las moscas blancas (Homoptera: Aleyrodidae) en América Central y el Caribe. CATIE. Turrialba, Costa Rica, pp. 64-66. 